05,11

\title{
Структурные особенности возникновения ферромагнитного порядка в системе $\mathrm{Mn}_{1-x} \mathrm{Cr}_{x} \mathrm{NiGe}$
}

\author{
() В.И. Вальков ${ }^{1}$, В.И. Каменев ${ }^{1}$, В.И. Митюк ${ }^{2}$, И.Ф. Грибанов ${ }^{1}$, \\ А.В. Головчан ${ }^{1}$, Т.Ю. Деликатная ${ }^{1}$ \\ ${ }^{1}$ Донецкий фризико-технический институт им. А.А. Галкина, \\ Донецк, Украина \\ ${ }^{2}$ НПЦ НАН Беларуси по материаловедению, \\ Минск, Беларусь \\ E-mail: valkov09@gmail.com \\ (Поступила в Редакцию 23 мая 2016 г. \\ В окончательной редакции 25 июня 2016 г.)
}

В рамках феноменологической модели взаимодействующих параметров магнитного и структурного порядков проведен анализ магнитных и структурных переходов в магнитокалорических сплавах системы $\mathrm{Mn}_{1-x} \mathrm{Cr}_{x} \mathrm{NiGe}$. На основе рассчитанных изобарических температурных зависимостей параметров магнитного и структурного порядков предсказано и подтверждено экспериментально скачок магнитной восприимчивости в области структурного перехода первого рода, обосновано изменение рода магнитного упорядочения при сближении магнитного и структурного переходов. Дано объяснение изменению рода фазового перехода при реверсивном изменении температуры и магнитного поля, которое наблюдается в ряде образцов исследуемой системы. Дан анализ эффективности использования индуцированных магнитным полем переходов для магнитокалорических приложений.

DOI: $10.21883 /$ FTT.2017.02.44046.202

\section{1. Введение}

Материалы с магнитоструктурными фазовыми переходами (МФП), в которых кристаллическая и магнитная симметрия изменяются при совпадающей или близких температурах, привлекают значительное внимание исследователей из-за существенных особенностей этих переходов. Эти особенности представляют интерес как для физики магнитных явлений, так и для практических приложений. Так, гигантский магнитокалорический эффект (МКЭ), обусловленный изменением энтропии при адиабатическом размагничивании (намагничивании) материала вблизи температуры магнитного фазового перехода, должен усиливаться, если возникновение или изменение магнитоупорядоченной фазы под действием магнитного поля сопровождается изменением симметрии или объема кристаллической ячейки [1,2]. Это очевидно, поскольку изменение магнитной части энтропии материала дополняется решеточными вкладами (структурно-симметрийным и объемным). Очевидно также, что для индуцированных магнитным полем МФП переходов порядок-порядок эти вклады могут быть определяющими, если степень магнитного беспорядка в конкурирующих фазах не нарушается при переходе, или, что то же, магнитные части энтропии этих фаз близки.

Настоящая работа посвящена теоретическому анализу экспериментальных исследований эволюции магнитных и структурных переходов в магнитокалорических сплавах системы $\mathrm{Mn}_{1-x} \mathrm{Cr}_{x} \mathrm{NiGe}$, в которой возможно не только сближение высокотемпературного пара- магнитного $(P M)$ структурного перехода первого рода типа смещения $[P M(63 / m m c)-P M(P n m a)]$ и низкотемпературного изоструктурного магнитного перехода второго рода парамагнитнетизм-ферромагнетизм $(P M($ Pnma $)-F M($ Pnma $))$, но их совмещение, приводящее к реализация магнитоструктурного перехода первого рода $P M(P 63 / m m c)-F M(P n m a)$. Низкотемпературные магнитные фазовые переходы порядок-порядок, обусловленные конкуренцией ферромагнитного и антиферромагнитного порядков, остаются за рамками настоящего рассмотрения.

\section{2. Эволюция магнитных и структурных переходов в системе $\mathrm{Mn}_{1-x} \mathrm{Cr}_{x} \mathrm{NiGe}$ в рамках модели взаимодействующих параметров структурного и магнитного порядков}

Для феноменологического описания магнитных и структурных переходов в рассматриваемой модели используем два параметра порядка, в качестве которых выбраны безразмерные величины $m$ и $\varphi$. Эти величины соответствуют относительным значениям компонент неприводимого вектора ферромагнетизма М и $x$-компоненты неприводимого вектора смещения атомов марганца $\mathbf{g}$ из соответствующих наборов неприводимых векторов (1) для элементарной кристаллохимической ячейки исходной гексагональной парамагнитной 
фазы $-P M(P 63 / m m c)[3]$, рис. 1

$$
\begin{gathered}
m=M_{x} / M_{0}, \\
\varphi= \pm\left|g_{x}\right| / 4 c_{\text {orth }}, \\
\mathbf{M}=\mathbf{M}_{1}+\mathbf{M}_{2}+\mathbf{M}_{3}+\mathbf{M}_{4}, \\
\mathbf{g}=\mathbf{u}_{\mathbf{1}}-\mathbf{u}_{2}-\mathbf{u}_{3}+\mathbf{u}_{4},
\end{gathered}
$$

где $M_{0}=\left|\mathbf{M}_{1}\right|+\mathbf{M}_{2}\left|+\mathbf{M}_{3}\right|+\left|\mathbf{M}_{4}\right|$ и $\left|\mathbf{M}_{i}\right|$ - магнитный момент насыщения $i$-той магнитной подрешетки марганца (вкладами в полный магнитный момент кристаллохимической ячейки от подрешеток $\mathrm{Ni}$ и Ge пренебрегается); $\mathbf{u}_{i}-$ смещение атомов марганца $i$ подрешетки при структурном переходе $P M(P 63 / m m c)-P M($ Рпта $)$, рис. 1. На рис. 1 гексагональная ячейка выбрана в ромбической установке (изображена жирными линиями, соединяющими атомы марганца $2-2-2-2$, где цифры обозначают номера позиций атомов марганца). Легко видеть, что структурный переход, обусловленный смещениями атомов $\mathrm{Mn}$, приводит к удвоению периода в базисной плоскости элементарной гексагональной ячейки (тонкие линии 1-1-2-2) вследствие нарушения пространственной эквивалентности атомов в позициях 1 и 2,3 и 4.

Выражение неравновесного термодинамического потенциала (НТП) деформируемой системы с двумя взаимодействующими параметрами порядка представим в виде суммы слагаемых, инвариантных относительно гексагональной группы симметрии $P 63 / m m c$

$$
\begin{aligned}
\Omega(m, \varphi, u)= & \Omega(m, u)+\Omega(\varphi, u)+\Omega(m, \varphi)+\Omega(u), \\
\Omega(m, u)= & \frac{a_{1}}{2}\left(T-T_{c}\right) m^{2}+\frac{1}{4} a_{3} m^{4}+\frac{1}{6} a_{5} m^{6} \\
& +\frac{1}{8} a_{7} m^{8}-m M_{0} H_{0}+T_{c} \frac{a_{1}}{2} \beta_{1} \omega m^{2}, \\
\Omega(\varphi, u)= & \frac{b_{1}}{2}\left(T-T_{t 1}\right) \varphi^{2}+\frac{1}{4} b_{3} \varphi^{4}+\frac{1}{6} b_{5} m^{6} \\
& +\varphi^{2}\left[T_{c} \frac{a_{1}}{2} \beta_{3} \omega+\Lambda_{1}\left(u_{x x}-u_{y y}\right),\right] \\
\Omega(m, \varphi)=\frac{a_{1}}{2} T_{c}\left(\delta+\delta_{1} \omega\right) m^{2} \varphi^{2} & \\
\Omega(u)= & \frac{1}{2 \kappa} \omega^{2}+\frac{1}{2} k_{1}\left(u_{x x}-u_{y y}\right)^{2}+P \omega,
\end{aligned}
$$

Выражение (2) является модифицированным выражением НТП Ф, которое было использовано в [4] для описания структурных $P M(P 63 / m m c) \leftrightarrow P M(P n m a)-$ и магнитоструктурных $P M(P n m a) \leftrightarrow F M(P 63 / m m c)$ переходов в MnAs. B [4] в качестве независимых упругих деформаций $u_{i}$ выбраны три комбинации тензора упругих деформаций $u_{z z}, u_{x x}-u_{y y}, u_{l l}=\omega$. Из условия равновесия $\partial \Phi / \partial u_{z z}=0$ компоненту $u_{z z}$ можно выразить через две оставшиеся. В этом случае для пространственно однородных параметров порядка и в пренебрежении коэффициентами термического расширения выражение $\Phi+\frac{a_{1}}{2} T_{c}\left(\delta_{1} \omega\right) m^{2} \varphi$ приводится к виду (2).

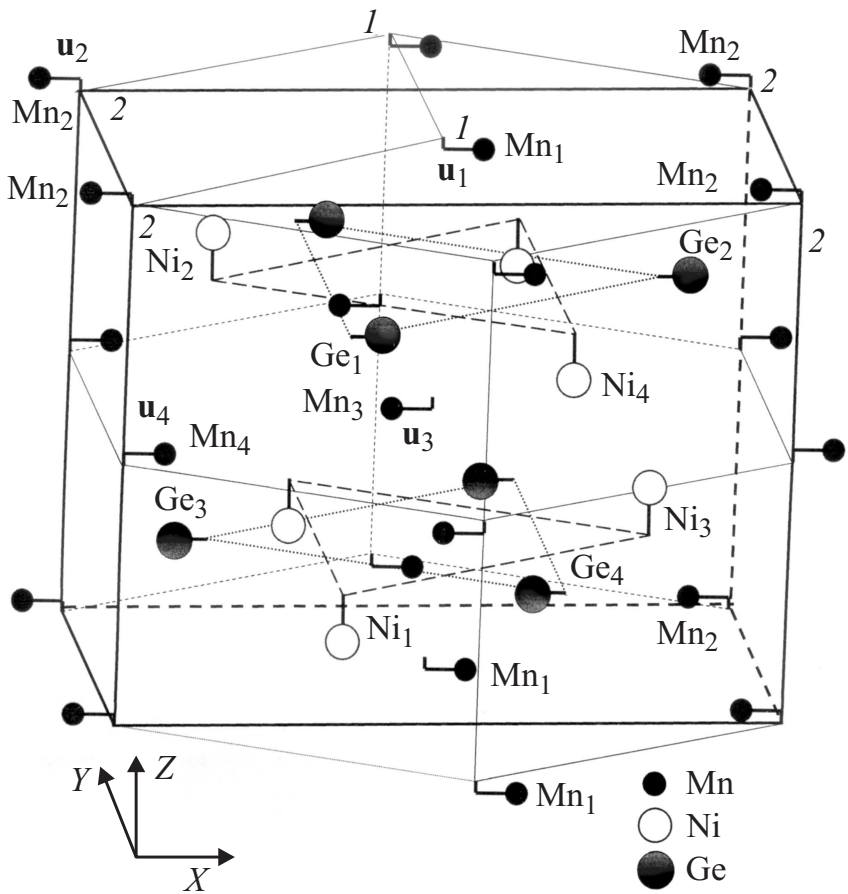

Рис. 1. Элементарная кристаллическая ячейка $\mathrm{MnNiGe}$ в ромбической установке. Параметры ячейки (толстые линии) определены так, что $c\left\|x, b_{\text {orth }}\right\| a_{\text {hex }}\left\|y, a_{\text {orh }}\right\| c_{\text {hex }} \| z$; смещения атомов марганца при переходе $(P 63 / m m c)-(P n m a)$ описываются векторами $\mathbf{u}$.

Слагаемое (2a) представляет НТП деформируемой системы, в которой может происходить изоструктурный переход $P M(m=0, \varphi=0) \leftrightarrow F M(m \neq 0, \varphi=0)$, соответствующий магнитному упорядочению $P M(P 63 / m m c) \leftrightarrow F M(P 63 / m m c)$ в гексагональной фазе $P 63 / m m c$; слагаемое $(2 \mathrm{~b})$ в НТП служит для описания структурного перехода $P M(\varphi=0) \leftrightarrow P M(\varphi \neq 0)$, который соответствует парамагнитному структурному переходу типа смещения $P M(P 63 / m m c) \leftrightarrow P M(P n т а)$. Взаимодействие между параметрами магнитного и структурного порядков описывается магнито-структурным вкладом (2c). Слагаемое (2d) описывает упругую энергию в гексагональной фазе $(\varphi=0)$ при использовании двух независимых комбинаций тензора упругих деформаций $u_{i k} ; \kappa=\left(k_{0}-\left(k_{0 z}\right)^{2} / 4 k_{z}\right)^{-1}-$ объемная сжимаемость в гексагональной фазе; $\beta_{i}\left(\lambda_{k}, k_{i}\right)$, $\delta_{i}\left(\lambda_{i}, k_{i}\right)$ - перенормированные постоянные, описывающие взаимодействия упругих деформаций с параметрами магнитного и структурного порядков; $k_{0}, k_{z}, k_{1}, k_{0 z}$ исходные упругие модули в выражении НТП при учете трех компонент $u_{i k} ; \lambda_{0}, \lambda_{z}, \lambda, \lambda_{1}, \Lambda_{0}, \Lambda_{z}, \Lambda_{1}$, - исходные магнитоупругие постоянные для НТП, описанного в [4].

Слагаемое $\Lambda_{1}\left(u_{x x}-u_{y y}\right) \varphi^{2} / 2$ в (2b), описывающее взаимодействие между структурным параметром порядка и орторомбическими искажениями $\left(u_{x x}-u_{y y}\right)$ гексагональной решетки как целого, ответственно за нарушение соотношения $c_{\text {orth }} \sqrt{3}=0$ (рис. 1) при возникновении смещений $\varphi$. 
Коэффициенты при $m^{2 n}$ в (2а) приближенно можно оценить при использовании перенормированной спиновыми флуктуациями плотности электронных состояний [3]. В этом случае коэффициенту при $m^{2}$ можно сопоставить обратную восприимчивость $X_{h e x}^{-1}$ системы взаимодействующих электронов в гексагональной решетке, а $T_{c}=\theta_{\text {hex }}^{*}$ соответствует парамагнитной температуре Кюри в недеформированной гексагональной фазе. Температурный коэффициент $\varphi^{2}$ при в (2b) связан с температурным размягчением одной из мягких мод, приводящей при $T=T_{t 1}$ к парамагнитному структурному переходу $P M(P 63 / m m c) \rightarrow P M(P n m a)$ типа смещения. Предполагается, что, как и в MnAs, этот структурный переход обусловлен понижением энергии электронной подсистемы из-за возникновения новых границ зоны Бриллюэна вследствие удвоения периода ячейки [3]. Последнее обстоятельство приводит к возникновению существенных аномалий в плотности электронных состояний, что может быть причиной изменений таких макроскопических величин, как объем, магнитный момент, магнитная восприимчивость [3,5,6], на фоне незначительных величин смещений $u_{i x} \propto \varphi$, которые характеризуют структурный переход.

Уравнения состояния (3) позволяют определить выражение для определения равновесных значений величин $\omega, u_{x x}-u_{y y}, \varphi, m$, оценить знаки коэффициентов $\beta_{1}$, $\beta_{3}, \delta, \delta_{1}$ и построить температурные и полевые зависимости параметров магнитного и структурного порядков

$$
\begin{gathered}
\partial \Omega / \partial \omega=0, \quad \partial \Omega / \partial\left(u_{x x}-u_{y y}\right)=0 \\
\partial \Omega / \partial \varphi=0, \quad \partial \Omega / \partial m=0 \\
\omega=-P \kappa-\frac{a_{1}}{2} T_{c} \beta_{1} \kappa m^{2}-\frac{a_{1}}{2} T_{c} \beta_{3} \kappa \varphi^{2}-\frac{a_{1}}{2} T_{c} \delta_{1} \kappa \varphi^{2} m^{2} \\
u_{x x}-u_{y y}=-\Lambda_{1} \varphi^{2} / 2 k_{1} \\
b_{1}\left(T-T_{1}\right)-T_{c} a_{1}\left(\delta_{1} m^{2}+\beta_{3}\right) P \kappa+\varphi^{2}\left(b_{3}-\Lambda_{1}^{2} / 2 k_{1}\right. \\
\left.-T_{c}^{2} \kappa a_{1}^{2}\left(\delta_{1} m^{2}+\beta_{3}\right)^{2} / 2\right)+b_{5} \varphi^{4}=0 \\
H M_{0}=a_{7} m^{7}+a_{5} m^{5}+a_{3} m^{3}+T_{c} a_{1}\left(\delta+\delta_{1} \omega\right) \varphi^{2} m \\
+T_{c} a_{1} m\left(\beta_{1} \omega\right)+a_{1}\left(T-T_{c}\right) .
\end{gathered}
$$

Очевидно, что если $T_{c}<T_{t 1}$, то при понижении температуры первым реализуется парамагнитный структурный переход типа смещения $P M(m=0, \varphi=0) \rightarrow$ $\rightarrow P M(m=0, \varphi \neq 0)$. При этом, поскольку в системе $\mathrm{Mn}_{1-x} \mathrm{Cr}_{x} \mathrm{NiGe}$, как и у большинства родственных германидов, этот переход является переходом первого рода и сопровождается увеличением объема [1], то

$$
\left(b_{3}-T_{c}^{2} a_{1}^{2} \kappa \beta_{3}^{2} / 2-\Lambda_{1}^{2} / 2 k_{1}\right)<0 \quad \text { и } \quad \beta_{3}<0 .
$$

Для магнитной подсистемы, в которой установление магнитного порядка в гексагональной фазе при $T_{c}(P)=\theta^{*}(P)=T_{c}+\beta_{1} \kappa P$ является переходом второго рода, и $T_{c}(P)$ не зависит от давления [7-12], потребуем выполнения соотношений

$$
\left(a_{3}-a_{1}^{2} \kappa \beta_{1}^{2}\right)>0, \quad \beta_{1}=0 .
$$

Уравнение (7), полученное из условия $\partial \Omega / \partial m=0$, позволяет также определить выражение для температурной зависимости обратной парамагнитной восприимчивости при $H=0$

$$
\begin{aligned}
\left(\frac{\partial H M_{0}}{\partial m}\right)_{H=m=0} & \equiv X^{-1}(T) \\
& =X_{h e x}^{-1}(T)+T_{c} a_{1}\left(\delta+\delta_{1} \omega_{m=0}\right) \varphi_{m=0}^{2} \\
& \equiv X_{h e x}^{-1}(T)+\Delta X_{\varphi}^{-1}(T)
\end{aligned}
$$

где $X_{h e x}^{-1}(T)=a_{1}\left(T_{c} \beta_{1} \omega_{m=0, \varphi=0}+T-\theta_{\text {hex }}^{*}\right)$ и $\Delta X_{\varphi}^{-1}(T)=$ $=T_{c} a_{1}\left(\delta+\delta_{1} \omega_{m=0}\right) \varphi_{m=0}^{2}-$ обратная $P M$ восприимчивость в гексагональной фазе $(\varphi=0)$ и добавка в орторомбической фазе $\varphi \neq 0$ соответственно.

Таким образом, модель предсказывает скачок обратной $P M$ восприимчивости при возникновении орторомбической структуры. Это позволяет фиксировать $P M$-структурный переход методами магнитометрии.

\section{3. Анализ решений уравнений состояния}

Решения уравнений состояния будем искать для постоянного соотношения $T_{t 1} / T_{c}=\tau_{t 1}=2.0$ и заданных значений упругих и магнитоупругих коэффициентов, удовлетворяющих неравенствам (8) и (9)

$$
\delta<0, \quad \delta_{1}>0 .
$$

Результатом решений являются зависимости $m(\tau)$, $\varphi_{0}(\tau)$ от безразмерной температуры $\tau=T / T_{c}$ при постоянных безразмерных давлениях $p=P / a_{1} T_{c}$, где $\varphi_{0}(\tau)=\sqrt{b_{1} / a_{1}} \varphi(\tau)$. На основе этих зависимостей построена модельная $p-\tau$-диаграмма, представленная на рис. 2.

Как видно из рис. 2, модельная $p-\tau$-диаграмма качественно сходна с известными экспериментальными $P-T$-диаграммами ряда германидов переходных металлов [7-10]. В частности, с $P-T$-диаграммой $\mathrm{MnNi}_{0.5} \mathrm{Co}_{0.5} \mathrm{Ge}$ (вставка на рис. 2).

Для модельной $p-\tau$-диаграммы существует 4 области характерных давлений.

При $0 \leq p \leq p_{1}$ последовательность зависимостей $m(\tau), \varphi_{0}(\tau)$ и характер магнитных и структурных переходов описываются кривыми 1,2 на рис. 3,a. Эти расчетные зависимости предсказывают ряд эффектов, которые легко проверить на эксперименте. Анализ этих зависимостей показывает, что структурный парамагнитный переход первого рода $P M(m=0, \varphi=0) \leftrightarrow P M(m=0, \varphi \neq 0)$ сопровождается температурным гистерезисом $\Delta \tau=\tau_{t 2}-\tau_{t 1}$ и скачкообразными изменениями параметра структурного 

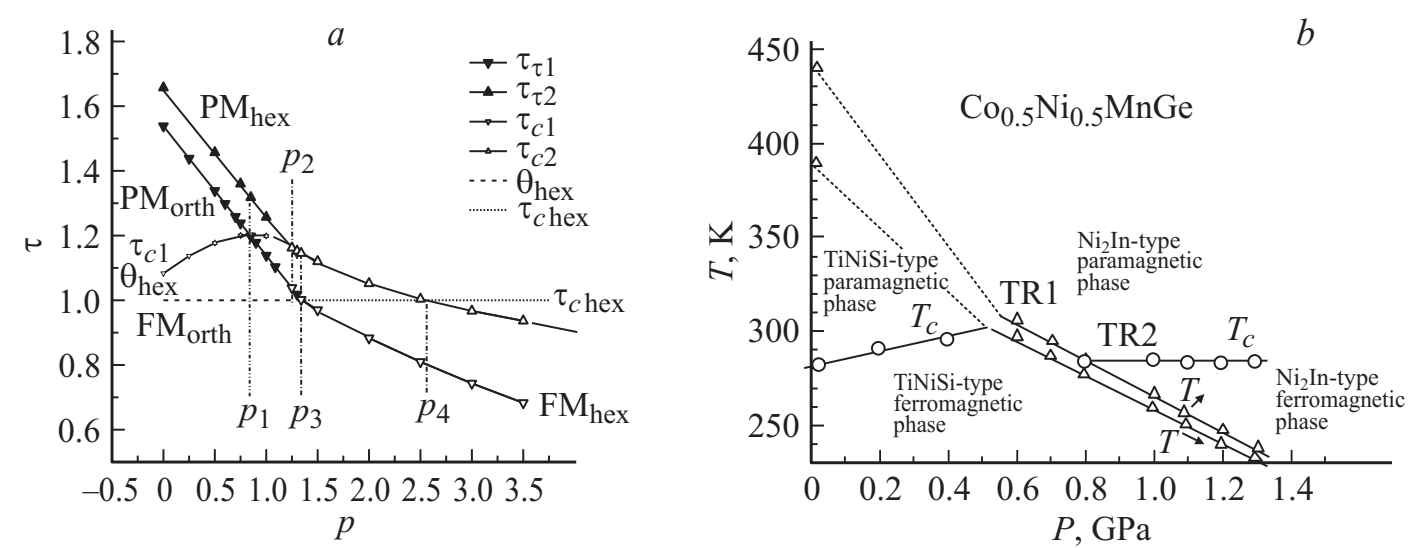

Рис. 2. Сопоставление теоретической $p-\tau$ и экспериментальной $P-T$-фазовых диаграмм. $a-$ теоретическая фазовая $p-\tau$-диаграмма (безразмерные единицы) в модели взаимодействующих параметров магнитного и структурного порядков; $b$ - экспериментальная $P-T$-диаграмма $\mathrm{Co}_{0.5} \mathrm{Ni}_{0.5} \mathrm{MnGe}$ из [9].
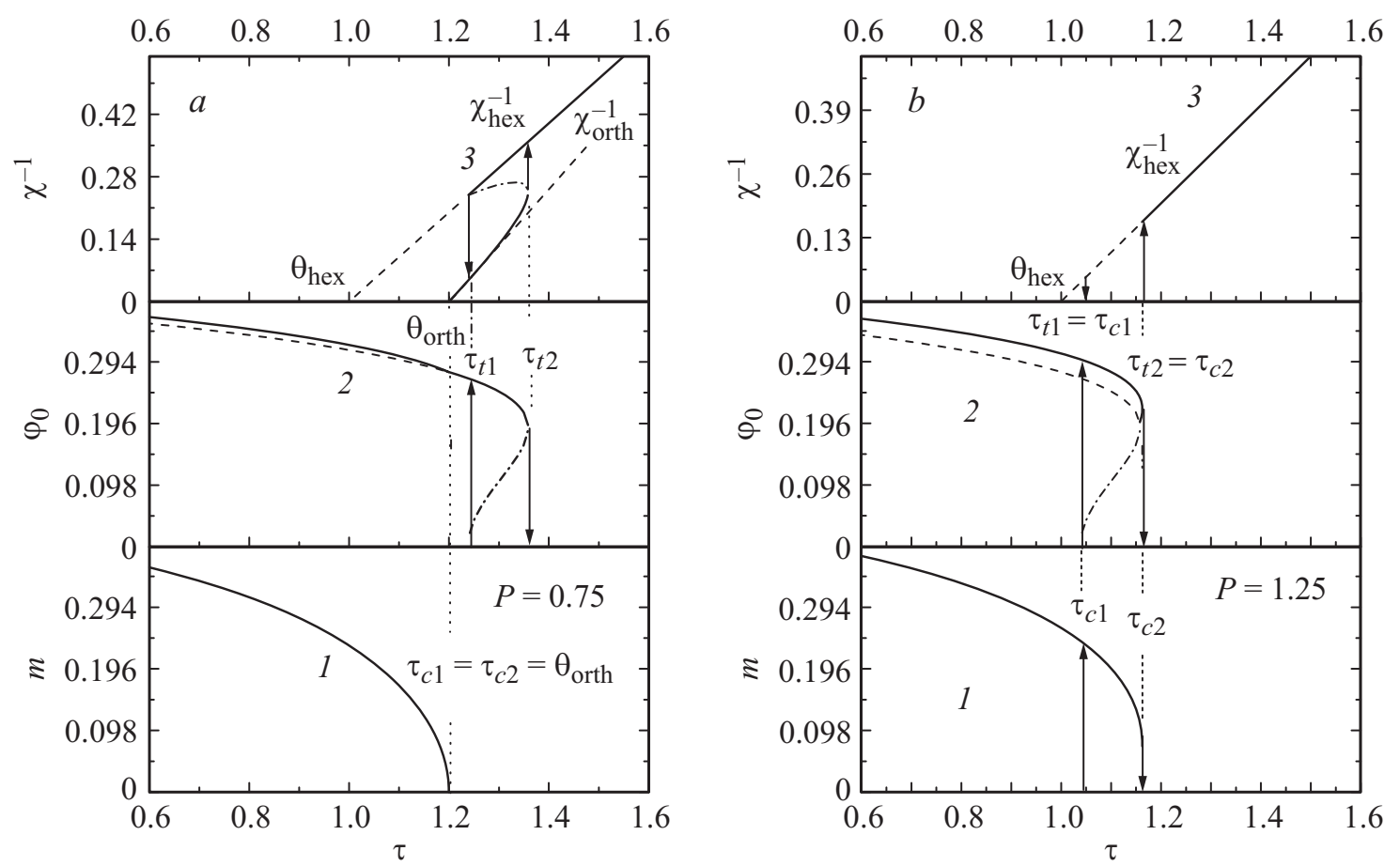

Рис. 3. Эволюция магнитных и структурных переходов в модели взаимодействующих параметров структурного и магнитного порядков по мере увеличения давления: $a: p=0.75 ; b: p=1.25$.

порядка $\varphi_{0}(\tau)$ и объема $\omega$. Возникновение $F M$ порядка при $\tau_{c 1}<\tau_{t 1}$ является изоструктурным переходом 2-го рода $P M(m=0, \varphi \neq 0) \rightarrow F M(m \neq 0, \varphi \neq 0)$. Температура Кюри $\tau_{c 1}$, которая находится из совместного решения уравнений $\left.(\partial \Omega / \partial \varphi)_{m=0}=0,(\partial \Omega / \partial m)_{m=0}=0\right)$, совпадает с парамагнитной температурой Кюри орторомбической фазы $\theta_{\text {orth }} \equiv \theta_{\text {orth }}^{*} / T_{c}$ и превосходит задаваемую изначально температуру Кюри гексагональной фазы $\tau_{c}=\theta_{\text {hex }}$. Значения величин $\theta_{\text {hex }}=\theta_{\text {hex }}^{*} / T_{c}$ и $\theta_{\text {orth }}$ находятся из экстраполяции к нулю зависимостей $\chi_{\text {hex }}^{-1}(\tau)=X_{\text {hex }}^{-1}(T) / a_{1} T_{c}$ и $\chi_{\text {orth }}^{-1}(\tau)=\chi_{\text {hex }}^{-1}(\tau)$ $+\Delta X_{\varphi}^{-1}(T) / a_{1} T_{c}$, как показано на кривой 3 рис. $3, a$. На этом и последующих графиках расчетные значения
$X^{-1}(T) / a_{1} T_{c} \equiv \chi^{-1}$ теоретических зависимостей $\chi^{-1}(\tau)$ указаны в безразмерных единицах.

Таким образом $\tau_{c 1}=\theta_{\text {orth }}$ определяет характеристическую температуру структурно-индуцированного $F M$-состояния. При этом ее значение и знак $\partial \tau_{c 1} / \partial p>0$ при заданых значениях величин $\beta_{3}<0, \beta_{1}=0$ определяются только значениями и знаками коэффициентов $\delta, \delta_{1}$, характеризующих силу взаимодействия между параметрами магнитного и структурного порядков. Поэтому при $\tau<\tau_{c 1}\left(\delta, \delta_{1}\right)$ формируется не только зависимость $m(\tau)$, но и новая зависимость $\varphi_{0}(\tau)$, которая проходит над парамагнитной зависимостью $\varphi_{0}(\tau)$ (штриховая линия на кривой 2 рис. $3, a)$. 


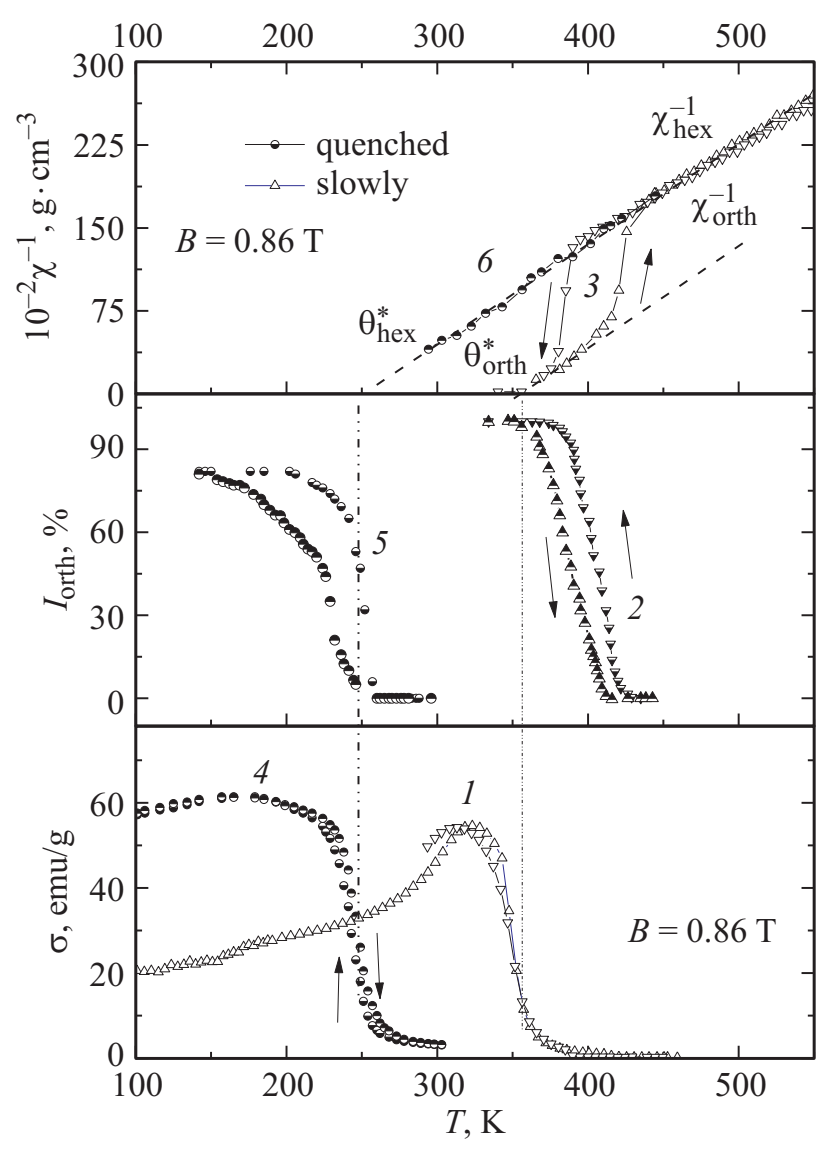

Рис. 4. Экспериментальные температурные зависимости основных магнитных и структурных характеристик для медленно охлажденного $(1,2,3)$ и быстрозакаленного $(4,5,6)$ образцов $\mathrm{Mn}_{0.89} \mathrm{Cr}_{0.11} \mathrm{NiGe}$.

Штрихпунктирные линии на этом и последующих рисунках соответствуют неравновесным решениям уравнений состояния (3), которые всегда присутствуют при описании переходов первого рода и соответствуют максимуму НТП.

Рассмотренное поведение характерных температурных зависимостей может кардинально измениться при смещении $P M$-структурного перехода к низким температурам. В нашей модели это достигается в области $p_{2} \leq p \leq p_{3}$. На рис. $3, b$ соответствующие зависимости (кривые $1,2,3)$ приведены для $p=1.25$. Как видно из рисунка, в этом случае магнитное разупорядочение становится магнитоструктурным переходом первого рода (кривая 1$)$, границы лабильности которого $\left(\tau_{c 1}, \tau_{c 2}\right)$ совпадают с границами лабильности $P M$-структурного перехода $\left(\tau_{t 1}, \tau_{t 2}\right)$ (кривая 2$)$.

Подобное поведение теоретических зависимостей можно сопоставить с характерными экспериментальными зависимостями для медленно охлажденного и закаленного от $800^{\circ} \mathrm{C}$ в воду образцов $\mathrm{Mn}_{0.89} \mathrm{Cr}_{0.11} \mathrm{NiGe}$ (рис. 4). Измерения намагниченнности проводились на стандартном магнитометре Vibrating Sample Magnetometer (VSM) фирмы Cryogenic Limited. Высокотемпературные измерения магнитной восприим- чивости проведены на оригинальном магнитометре, описанном в [11].

Из сравнения рисунков видно, что предсказанные в теории аномалия обратной восприимчивости и ее разделение на гексагональную и орторомбическую ветвь подтверждаются. Это позволяет выделить из экспериментальной кривой 3 на рис. 4 зависимости $\chi_{\text {hex }}^{-1}(T)$ и $\chi_{\text {orth }}^{-1}(T)$, из которых можно определить эффективные парамагнитные моменты на атом для гексагональной $\left(p_{\text {hex }}^{e f} \approx 4.1 \mu_{B}\right)$ и $p_{\text {orth }}^{e f} \approx 4.1 \mu_{B}$ орторомбической фаз.

На рис. 4 (кривая 2) приведена температурная зависимость содержания ромбической фазы в образце $I_{\text {orth }}(T)$ в процессе перехода, которая определялась методом рентгенофазового анализа по изменению интенсивностей дифракционных максимумов сменяющихся фаз с температурой. Использовалось $\mathrm{Cu} K_{\alpha}$-излучение. Этим зависимостям могут быть сопоставлены расчетные зависимости $\varphi_{0}(\tau)$ и $\chi^{-1}(\tau)$ (кривые 2,3 на рис. $3, a)$. Изоструктурному магнитному фазовому переходу 2-го рода $P M(P n m a) \leftrightarrow F M(P n m a)$, который описывается безгистерезисной зависимостью $\sigma(T)$ (кривая 1 на рис. 4), может быть сопоставлена модельная зависимостью, которая описывает переход второго рода $P M(m=0, \varphi \neq 0) \leftrightarrow F M(m \neq 0, \varphi \neq 0)$ (кривая 1 на рис. $3, a)$.

Аналогичные модельные зависимости на рис. $3, b$ сопоставляются с экспериментальными температурными зависимостями 4, 5, 6 закаленного образца $\mathrm{Mn}_{0.89} \mathrm{Cr}_{0.11} \mathrm{NiGe}$ (рис. 4). Сравнение и анализ этих зависимостей показывает, что реализация ферромагнитного упорядочения как магнитоструктурного перехода первого рода $P M(P 63 / m m c) \leftrightarrow F M(P n m a)$ происходит в результате сближения и совмещения температур магнитного и структурного переходов. Это совмещение может быть достигнуто различными способами: соответствующей закалкой образца [2] или наложением внешнего давления [9].

$\mathrm{B}$ области промежуточных давлений $p_{1} \leq p \leq p_{2}$ теоретические зависимости параметров порядка и обратной восприимчивости обнаруживают уникальное поведение. Из расчетных зависимостей $m(\tau), \varphi_{0}(\tau)$ следует, что возникновение магнитного порядка на линии $\tau_{c 1}(p)=\tau_{t 1}(p)$ является магнитоструктурным переходом первого рода $P M(m=0, \varphi=0) \rightarrow F M(m \neq 0, \varphi \neq 0)$ при $\partial \tau_{c 1} / \partial p=\partial \tau_{t 1} / \partial p<0$. Напротив, исчезновение магнитного порядка на линии $\tau_{c 2}(p)<\tau_{t 2}(p)$ является изоструктурным переходом второго рода $F M(m \neq 0, \varphi \neq 0) \rightarrow P M(m=0, \varphi \neq 0) . \quad$ При этом $\partial \tau_{c 2} / \partial p \geq 0$. Более детально этот случай иллюстрируется на рис. 5, $a$. Соответствующим модельным зависимостям на этом рисунке могут быть сопоставлены экспериментальные температурные зависимости намагниченности 1, концентрации орторомбической фазы 2 и обратной восприимчивости 3, измеренные на образце $\mathrm{Mn}_{0.82} \mathrm{Cr}_{0.18} \mathrm{NiGe}$, рис. 5, $b$. На этом рисунке видно, что возникновение $F M$-порядка при понижении температуры сопровождается относительно резким 

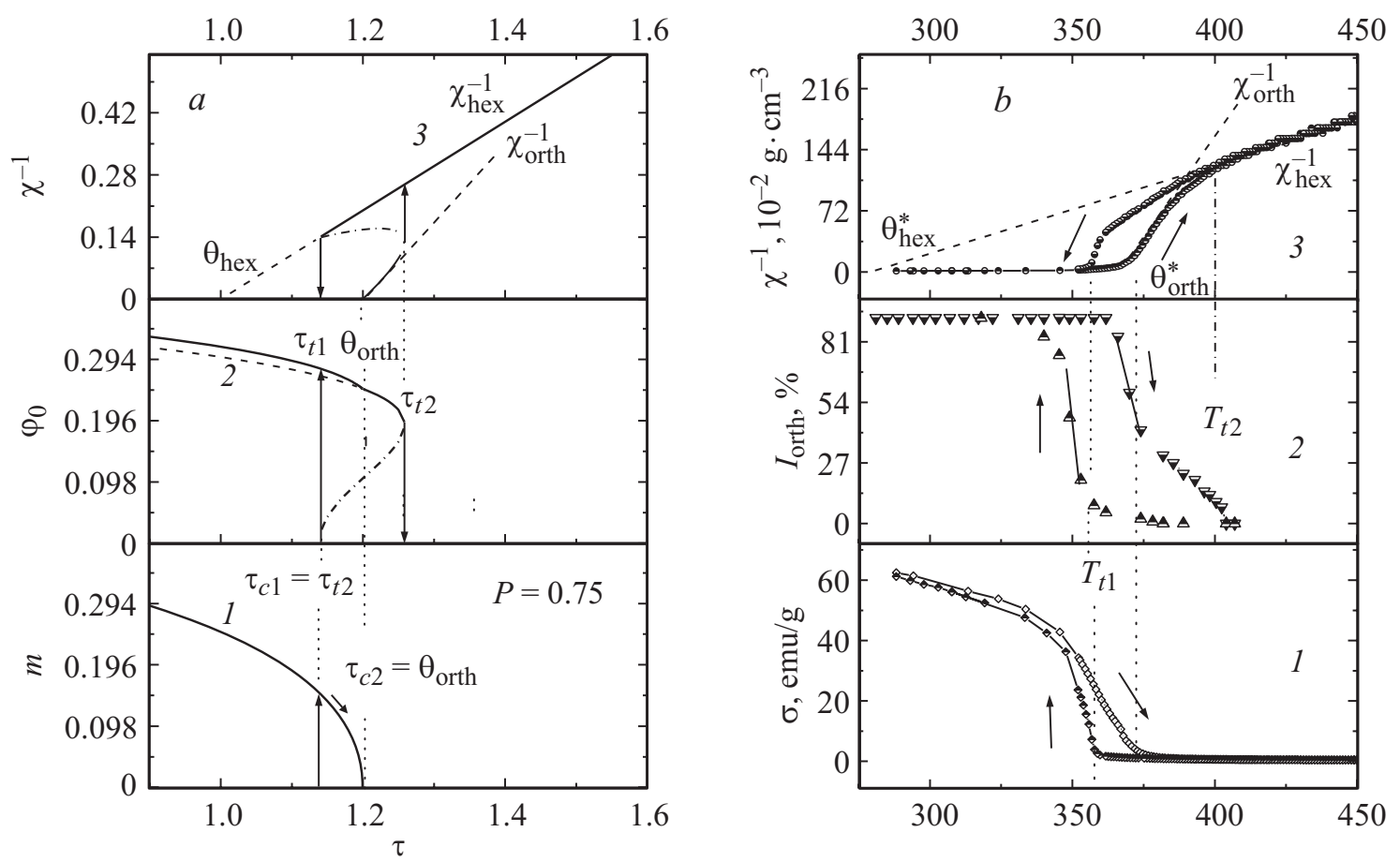

Рис. 5. Теоретическое обоснование $(a)$ и экспериментальное подтверждение $(b)$ сосуществования переходов 1-го и 2-го рода в системе $\mathrm{Mn}_{1-x} \mathrm{Cr}_{x} \mathrm{NiGe} . a-$ теоретические температурные зависимости рассчитаны для $p=1.0 ; b-$ экспериментальные температурные зависимости основных магнитных $(1,3)$ и рентгеноструктурных (2) характеристик образца $\mathrm{Mn}_{0.82} \mathrm{Cr}_{0.18} \mathrm{NiGe}$

изменением намагниченности (кривая $\sigma(T))$ и возникновением орторомбических искажений решетки (кривая 2) и поэтому может определяться как магнитоструктурный переход первого рода. Напротив, при последующем нагревании образца исчезновение $F M$ порядка описывается практически плавной функцией $\sigma(T)$ при температуре $T_{c 2}=\theta_{\text {orth }}$, не приводит к изменению симметрии решетки $\theta_{\text {orth }}<T_{t 2}$ и может характеризоваться как изоструктурный переход второго рода.

В области $p_{3}<p<p_{4}$ при понижении температуры вначале на линии $\theta_{\text {hex }}=(\tau)$ наблюдается изоструктурный переход второго рода $P M(m=0, \varphi=0) \rightarrow$ $\rightarrow F M\left(m_{1} \neq 0, \varphi=0\right)$. При более низких температуpax на линии $\tau_{c 1}(p)=\tau_{t 1}(p)$ происходит магнитоструктурный переход первого рода $P M\left(m_{1} \neq 0, \varphi=0\right) \rightarrow$ $\rightarrow F M\left(m_{2}>m_{1}, \varphi \neq 0\right)$. При последующем повышении температуры на линии $\tau_{c 2}(p)=\tau_{t 2}(p)>\theta_{h e x}$ наблюдается магнитоструктурный переход первого рода $F m\left(m_{2} \neq 0, \varphi \neq 0\right) \rightarrow P M(m=0, \varphi=0)$. Экспериментальные аналоги подобных переходов нам не известны.

При $p>p_{4}>$ наблюдается следующая последовательность магнитных фазовых переходов. На линии $\tau_{c h e x}=\theta_{\text {hex }}$ реализуется изоструктурный переход второго рода $P M(m=0, \varphi=0) \rightarrow F M\left(m_{1} \neq 0, \varphi=0\right)$, который сопровождается плавным возрастанием параметра магнитного порядка вплоть до значения $m=m_{1}$ (зависимость la на рис. 6). При более низких температурах на линии $\tau_{c 1}(p)=\tau_{t 1}(p)$ реализуется магнитоструктурный переход первого рода порядок-порядок
$F M\left(m_{1} \neq 0, \varphi=0\right) \rightarrow F M\left(m_{2}>m_{1}, \varphi \neq 0\right), \quad$ сопровождающийся резким увеличением параметра магнитного порядка от $m_{1}$ до $m_{2}$ и переходом от зависимости $1 a$ к зависимости $1 b$. Обратное повышение температуры приводит вначале к магнитоструктурному переходу первого рода $F M\left(m_{2} \neq, \varphi \neq 0\right) \rightarrow F M\left(m_{1}<m_{2}, \varphi=0\right)$ на линии $\tau_{c 2}(p)=\tau_{t 2}(p)$ и в последующем на линии $\tau_{c h e x}=\theta_{\text {hex }} \quad$ к изоструктурному магнитному переходу второго рода $F M\left(m_{1} \neq 0, \varphi=0\right) \rightarrow P M\left(m_{1}=0, \varphi=0\right)$. Для этого диапазона давлений зависимости $m(\tau), \varphi_{0}(\tau)$, $\chi^{-1}(\tau)$ приведены на рис. 6. При этом зависимость $m(\tau)$ можно сопоставить экспериментальной температурной зависимости для $(\mathrm{MnCo})_{0.97} \mathrm{Ge}$, измеренной в [12].

Поскольку $\mathrm{Mn}_{1-x} \mathrm{Cr}_{x} \mathrm{NiGe}$ является перспективной магнитокалорической системой, то практический интерес представляют теоретический анализ процессов намагничивания вблизи температуры магнитного разупорядочения. На рис. 7 представлены модельные полевые зависимости для трех наиболее характерных случаев, которые реализуются в процессе увеличения давления.

Для $p=0.75$, когда спонтанные переходы по температуре являются изоструктурными переходами второго рода, зависимости $m(h), \varphi_{0}(h) \quad\left(h=M_{0} H / a_{1} T_{c}\right)$ построены для двух температур. При $\tau \geq \tau_{c 1}=\tau_{c 2}<\tau_{t 1}$ исходное $P M$ состояние соответствует орторомбической фазе $\left(\left|\varphi_{0}(h)>0\right|\right)$, поэтому $\varphi_{0}(h)$ на этом рисунке не претерпевает значительных изменений и обе кривые $(1,2)$ описывают простой изоструктурный процесс намагничивания. Однако при $\tau \geq \tau_{t 2}>\tau_{c 1}$ $P M$ состоянию соответствует гексагональная фаза 


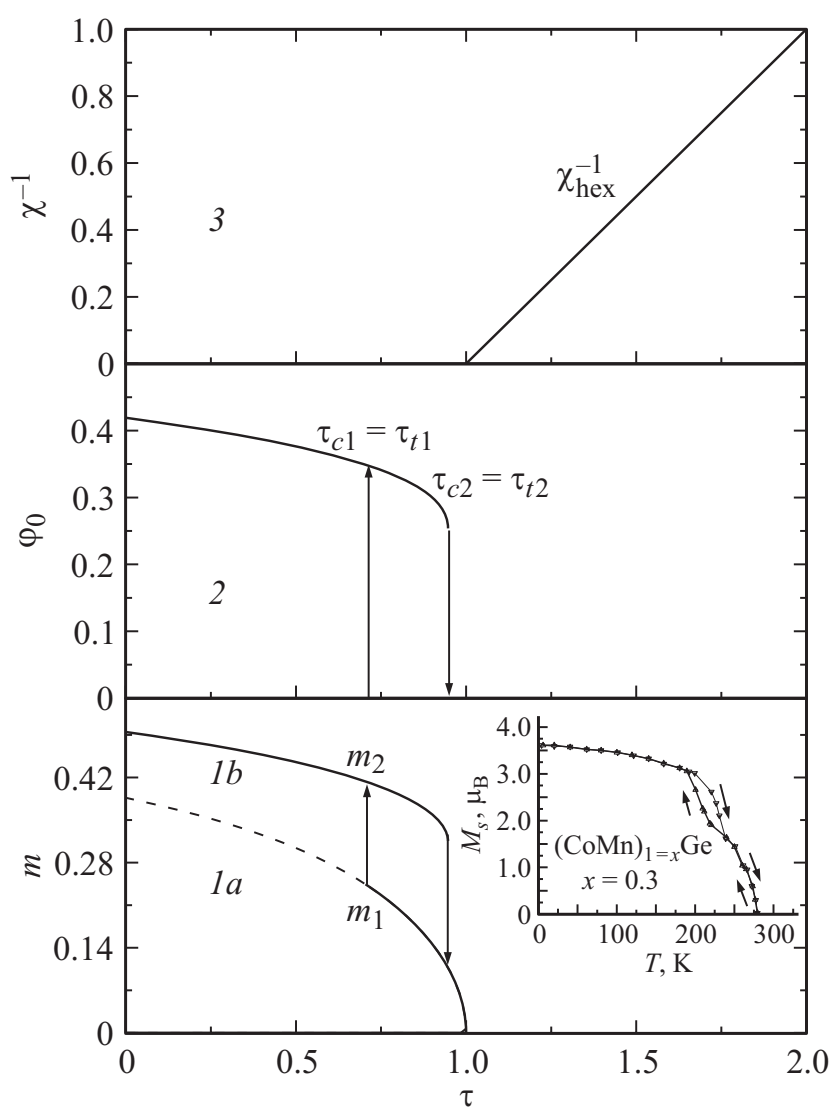

Рис. 6. Температурная последовательность магнитоструктурных упорядочений, рассчитанная для $p=3.3$. На вставке: экспериментальные данные, приведенные из [13] для $(\mathrm{CoMn})_{0.97} \mathrm{Ge}$.

$\left(\varphi_{0}(h=0)=0\right)$ и кривые намагничивания кардинально изменяются. Зависимости $m(h), \varphi_{0}(h)$ описывают в этом случае обратимые индуцированные магнитным полем магнитоструктурные переходы первого рода $P M(m=0, \varphi=0) \leftrightarrow F M\left(m_{1} \neq 0, \varphi \neq 0\right)(1 a, 2 a)$. Эти переходы стимулируются взаимосвязью между магнитным состоянием решетки и ее кристаллографической симметрией, которая описывается в НТП слагаемым (2c). Скачкообразное изменение параметра магнитного порядка $\Delta m_{1}\left(\Delta m_{2}\right)$ при $h=h_{k 1}\left(h=h_{k 2}\right)$ соответственно сопровождается изменением структурного параметра порядка $\Delta \varphi_{01}\left(\Delta \varphi_{02}\right)$ и как следствие дополнительным симметрийно-структурным вкладом, усиливающим полное изменение энтропии $\Delta S$ магнитополевого процесса.

Для магнитокалорических приложений эти переходы, однако, малоэффективны. Действительно в рассматриваемой модели $\Delta S$ определяется в основном суперпозицией двух однознаковых слагаемых (9) и при температурах $\tau \geq \tau_{t 2} \gg \tau_{c 1}$ изменением $\Delta m^{2}$ согласно рис. 6 можно пренебречь.

$$
\Delta S_{h=h_{k 1}}=-\frac{\partial \Delta \Omega}{\partial T}=-\frac{1}{2} a_{1} \Delta m^{2}-\frac{1}{2} b_{1} \Delta \varphi^{2} \approx-\frac{1}{2} b_{1} \Delta \varphi^{2} .
$$

В интервале давлений $p_{2} \leq p \leq p_{3}$, когда спонтанное магнитное разупорядочение (упорядочение) является магнитоструктурным переходом первого рода $P M(m=0, \varphi=0) \leftrightarrow F M\left(m_{1} \neq 0, \varphi \neq 0\right)$, возникает более благоприятная ситуация для магнитокалорических приложений. Действительно, полевые зависимости $m(h)$, $\varphi_{0}(h)$ (кривые 3,4 на рис. 7) уже при $\tau \geq \tau_{c 2}=\tau_{t 2}$ демонстрируют индуцированные магнитным полем обратимые магнитоструктурные переходы первого рода $P M(m=0, \varphi=0) \leftrightarrow F M(m \neq 0, \varphi \neq 0)$, сопровождающиеся четким разделением фаз и значительными изменениями параметров магнитного и структурного порядков. В этом случае оба слагаемых в (12) вносят существенный вклад в изменение энтропии. При атмосферном давлении такие переходы наблюдаются в закаленном образце $\mathrm{Mn}_{0.89} \mathrm{Cr}_{0.11} \mathrm{NiGe}$ (вставка на рис. 7).

Зависимости 4,5 на рис. 7 описывают процессы намагничивания для случая $p_{1}<p<p_{2}$ при $\tau<\tau_{c 2}<\tau_{t 2}$, когда согласно рис. 5, а спонтанные переходы при по-

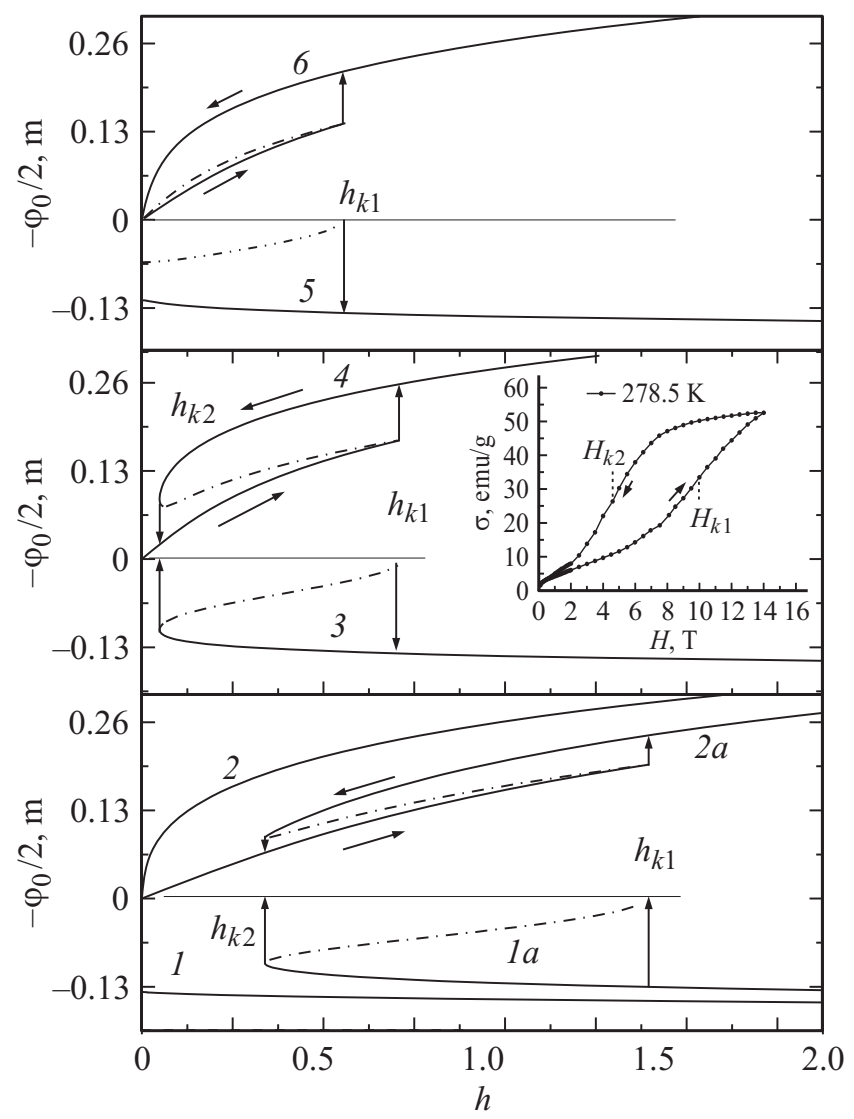

Рис. 7. Расчетные полевые зависимости параметров магнитного и структурного порядков для трех наиболее характерных случаев, которые реализуются в процессе увеличения давления. $(1,2)-p=0.75, \tau=1.23<\tau_{t 1} ;(1 a, 2 a)-p=0.75$, $\tau=1.38>\tau_{t 2} ;(3,4)-p=1, \tau \geq \tau_{c 2}=\tau_{t 2} ;(5,6)-p=1.25$, $\tau_{t 2}>\tau>\tau_{c 1}$; вертикальными стрелками вверх (вниз) обозначены значения величин $\Delta m_{1}\left(\Delta m_{2}\right)$ для $m(h)$ и $\Delta \varphi_{01}\left(\Delta \varphi_{02}\right)$ для $\varphi_{0}(h)$; на вставке: экспериментальная полевая зависимость намагниченности для закаленного образца $\mathrm{Mn}_{0.89} \mathrm{Cr}_{0.11} \mathrm{NiGe}$, измеренная в [2]. 
нижении температуры являются магнитоструктурными переходами первого рода, а при повышении температуры - изоструктурными переходами второго рода. Полевые зависимости $(4,5)$ также показывают нетривиальное поведение. При возрастании поля до значения первого критического $h_{k 1}$ обе зависимости $m(h), \varphi(h)$ описывают индуцированный полем магнитоструктурный переход первого рода $P M(m=0, \varphi=0) \rightarrow F M\left(m_{1} \neq 0, \varphi \neq 0\right)$, сопровождающийся скачкообразным возрастанием параметров порядка. При последующем снижении поля разделение фаз не происходит. Наблюдается только плавное изоструктурное (в пределах орторомбической симметрии) исчезновение намагниченности $F M(m>0, \varphi \neq 0) \rightarrow P M(m=0, \varphi \neq 0)$ (кривая 4). Экспериментальные аналоги полевых зависимостей намагниченности (кривые $1 a, 2 a$ и 4,5 на рис. 7), которые могут реализоваться в незакаленных образцах $\mathrm{Mn}_{0.89} \mathrm{Cr}_{0.11} \mathrm{NiGe}$ и $\mathrm{Mn}_{0.82} \mathrm{Cr}_{0.18} \mathrm{NiGe}$, на сегодняшний день не измерены из-за высоких характеристических температур $F M$ разупорядочения.

\section{4. Обсуждение}

Рассмотренная модель взаимодействующих параметров магнитного и структурного порядков обобщает и дополняет новыми результатами ранее предложенные в работах [4,9] модели. Например, в [9] для описания магнитоструктурных переходов в родственных германидах отсутствует такой важный результат, как скачок парамагнитной восприимчивости вследствие $P M$ структурного перехода первого рода. Также из теоретической $P-T$-диаграммы в [9] следует противоречащий эксперименту результат о понижении температуры изоструктурного перехода второго $P M(m=0, \varphi \neq 0)-F M(m \neq 0, \varphi \neq 0)$ с ростом давления. В рассматриваемой здесь модели, как видно из рис. 2, зависимость этой температуры $\left(\tau_{c 1}=\tau_{c 2}\right)$ от давления является возрастающей функцией давления $\left(\partial \tau_{c 1} / \partial p>0\right)$. Этот результат является следствием введения в термодинамический потенциал дополнительного инварианта $\frac{a_{1}}{2}\left(\delta_{1} \omega\right) m^{2} \varphi^{2}$, который напрямую учитывает взаимодействие параметров магнитного и структурного порядков с объемными деформациями и ,регулирует“ знак $\partial \tau_{c 1} / \partial p$. Действительно, для $\beta_{3}<0, \delta<0, \delta_{1} \leq 0$, $\partial \tau_{c 1} / \partial p<0$. При сохранении величин и знаков первых двух коэффициентов $\left(\beta_{3}<0, \delta<0\right)$ и изменении знака и величины только коэффициента $\delta_{1}$ можно получить $\partial \tau_{c 1} / \partial p>0$, если $\delta_{1}>\delta_{1 k}>0$.

В рассмотренной модели также было обосновано сосуществование спонтанных и индуцированных магнитным полем магнитоструктурного перехода первого рода при охлаждении (намагничивании) и изоструктурного перехода второго рода при нагревании (размагничивании), которые реализуются в образце $\mathrm{Mn}_{0.82} \mathrm{Cr}_{0.18} \mathrm{NiGe}$.

В заключение отметим две важные особенности, которые позволяют приблизиться к пониманию механизма магнитоструктурных переходов в исследуемой системе.
К первой можно отнести возрастание магнитного момента насыщения $\mu$, сопровождающее магнитоструктурный переход первого рода $F M\left(\mu_{\text {hex }}, \varphi=0\right) \rightarrow$ $\rightarrow F M\left(\mu_{\text {orth }}>\mu_{\text {hex }}, \varphi \neq 0\right)$, что экспериментально наблюдается в родственных германидах $(\mathrm{MnCo})_{1-x} \mathrm{Ge}$ при атмосферном давлении [13]. В системе $\mathrm{Mn}_{1-x} \mathrm{Cr}_{x} \mathrm{NiGe}$ наблюдение данной особенности предсказывается нашими расчетами для больших давлений (рис. 6).

Поскольку $\quad P_{\text {orth }}^{e f} / \mu_{\text {orth }} \equiv 4.0 \mu_{B} / 2.1 \mu_{B}>1$, то носителями магнетизма являются коллективизированные $d$-электронные состояния марганца, образующие $d$-зону [14]. Коллективизированный характер носителей магнетизма в германидах переходных металлов составляет вторую особенность этих материалов.

Далее, поскольку магнитный момент насыщения на атом в орторомбической фазе превосходит магнитный момент насыщения в гексагональной фазе $\left(\mu_{\text {orth }}>\mu_{\text {hex }}\right)$, то магнитоструктурный переход первого рода $F M\left(\mu_{\text {hex }}, \varphi=0\right) \rightarrow F M\left(\mu_{\text {orth }}, \varphi \neq 0\right)$, связанный с резким возрастанием локального магнитного момента Mn, может стимулироваться определенной трансформацией спин-поляризованной электронной структуры.

Действительно, увеличение локального магнитного момента Mn, образованного за счет обменного расщепления спиновых подзон $d$-электронных состояний марганца, может произойти путем увеличения этого обменного расщепления. А это возможно только через еще большее увеличение заселенности нижней по энергии антисвязующей $d$-зоны и большему опустошению верхней.

Очевидно, что энергетическая стабилизация антисвязующих электронных состояний приводит к „включению“ дополнительных связей между атомами в решетке, которые могут стать причиной изменения исходной (гексагональной) атомной конфигурации и перехода к новой (орторомбической).

\section{5. Заключение}

В рассмотренной феноменологической модели на основе теоретической $p-\tau$-диаграммы прослежены наиболее характерные особенности структурно-индуцированных переходов парамагнетизм-ферромагнетизм в сплавах системы $\mathrm{Mn}_{1-x} \mathrm{Cr}_{x} \mathrm{NiGe}$.

Показано, что появление структурно-индуцированного ферромагнетизма в исследуемой системе обусловлено взаимодействием между параметрами магнитного и структурного порядков в сопряжении с объемными деформациями.

Дано объяснение аномальному скачку парамагнитной восприимчивости в области парамагнитного структурного перехода первого рода.

Дано обоснование сосуществования в определенном интервале давлений $p-\tau$-диаграммы спонтанных и индуцированных магнитным полем магнитоструктурных фазовых переходов первого и второго рода. 


\section{Список литературы}

[1] N.T. Trung, V. Biharie, L. Zhang, L. Caron, K.H.J. Buschow, E. Brück. Appl. Phys. Lett. 96, 162507 (2010).

[2] А.П. Сиваченко, В.И. Митюк, В.И. Каменев, А.В. Головчан, В.И. Вальков, И.Ф. Грибанов. ФНТ 39, 1350 (2013).

[3] В.И. Вальков, А.В. Головчан. ФНТ 31, 695 (2005).

[4] С.К. Асадов, Э.А. Завадский, В.И. Каменев, Е.P. Stefanovskii, А.Л. Сукстанский, Б.М. Тодрис. ФТТ 42, 1649 (2000).

[5] J.-T. Wang, D.-S. Wang, C. Chen, O. Nashima, T. Kanomata, H. Mizuseki, Y. Kawazoe. Appl. Phys. Lett. 89, 262504 (2006).

[6] F. Guillou, F. Wilhelm, O. Tegus, A. Rogalev. arXiv:1602.08263v1 [cond-mat.str-el] 26 Feb 2016.

[7] S. Anzai, K. Ozaw. Phys. Rev. B 18, 2173 (1978).

[8] L. Caron, N.T. Trung, E. Brück. Phys. Rev. B 84, 020 414(R) (2011).

[9] J.S. Niziol, A. Zieba, R. Zach, M. Baj, L. Dmowski. JMMM 38, 205 (1983).

[10] T. Jaworska-Gołb, S. Baran, R. Duraj, M. Marzec, V. Dyakonov, A. Sivachenko, Yu. Tyvanchuk, H. Szymczak, A. Szytuła. JMMM 385, 6, (2015).

[11] Г.И. Маковецкий, А.И. Галяс, К.И. Янушкевич, В.И. Шамбалев, А.К. Богуш. Вестн. НАН Беларуси. Сер. физ.-техн. 3, 10 (2000).

[12] J.S. Niziol, R. Zach, J.P. Senateur, J. Beille. J MMM 79, 333 (1989).

[13] Н.В. Мушников. УФН 182, 450 (2012).

[14] Т. Мория. Спиновые флуктуации в магнетиках с коллективизированными электронами. Мир, Москва (1988). 288 с. 